International Mathematical Forum, 2, 2007, no. 55, 2701 - 2710

\title{
Group Invariant Solutions of Burgers-Poisson Equation
}

\author{
Nurettin Cenk Turgay \\ Department of Mathematics \\ Faculty of Science and Letters \\ Istanbul Technical University \\ 34369 Istanbul, Turkey \\ Emanullah Hizel ${ }^{1}$ \\ Department of Mathematics \\ Faculty of Science and Letters \\ Istanbul Technical University \\ 34369 Istanbul, Turkey \\ hizel@itu.edu.tr
}

\begin{abstract}
In this paper, a nonlinear dispersive wave equation Burgers-Poisson (BP) equation is considered. We present a classification of group invariant solutions for the BP equation by using classical Lie method.
\end{abstract}

Mathematics Subject Classification; 35Q53

Keywords: Integration, Lie algebras, Nonlinear Partial Differential Equations

\section{Introduction}

$\mathrm{BP}$ equation is a nonlinear dispersive wave equation given by

$$
u_{t}+u_{x}+u u_{x}-u_{x x t}-\left(3 u_{x} u_{x x}+u u_{x x x}\right)=0,
$$

where $u(x, t)$ is a function of the spatial coordinate $x$ and the time $t$, and the subscripts $t$ and $x$ denote partial differentiation. This equation has been

\footnotetext{
${ }^{1}$ Corresponding author
} 
proposed to describe the unidirectional propagation of long waves in dispersive media $[1,2,3]$.

The application of Lie transformations group theory for the construction of solutions of nonlinear partial differential equations ( PDEs) is one of the most active fields of research in the theory of nonlinear PDEs and applications. The fundamental basis of the technique is that when a differential equation is invariant under a Lie group of transformations, a reduction transformation exists. In order to determine solutions of PDE (1) that are not equivalent by the action of group, we must calculate the one dimensional optimal system. Most of the required theory and description of the method can be found in $[4,5,6,7]$. The aim of this paper is to find the symmetry group of (1) and present a classification of its one-dimensional subgroup. By using this subgroup is to perform symmetry reduction and to obtain the group invariant solutions.

\section{Symmetry group analysis of BP equation}

\subsection{Lie symmetry generators}

In this subsection, we want to present the most general Lie group of point transformations, which leaves BP equation (1) invariant.

Definition 1. We consider a scalar $k$-th order PDE represented by

$$
\Delta\left(\sim_{\sim}^{x}, \underset{\sim}{u}, \sim_{1}^{u}, \ldots, \sim_{k}^{u}\right)=0
$$

$\underset{\sim}{x}=\left(x_{1}, x_{2}, \ldots, x_{n}\right)$ denotes $n$ independent variables, $\underset{\sim}{u}=\left(u^{1}, u^{2}, \ldots, u^{m}\right)$ denotes set of $m$ dependent (differential) variables, and $\underset{\sim}{u}$ denotes set of corresponding to all $j$ th-order partial derivatives of $\underset{\sim}{u}$ with respect to variable $\underset{\sim}{x}$. The infinitesimal generator of the one-parameter Lie group of transformations for equation (2) is

$$
X=\sum_{i=1}^{n} \xi_{i}(\underset{\sim}{x}, \underset{\sim}{u}) \frac{\partial}{\partial x_{i}}+\sum_{\alpha=1}^{m} \eta^{\alpha}(\underset{\sim}{x}, \underset{\sim}{u}) \frac{\partial}{\partial u^{\alpha}}
$$

where $\xi_{i}(\underset{\sim}{x}, \underset{\sim}{u}), \eta^{\alpha}(\underset{\sim}{x}, \underset{\sim}{u})$ are the infinitesimals $(3)$, and the $k$ th prolongation of the infinitesimal generator (3) [4-6] is

$$
p r^{(k)} X=X+\eta_{i}^{(1) \alpha}(\underset{\sim}{x}, \underset{\sim}{u}, \underset{\sim}{u}) \frac{\partial}{\partial u_{i}^{\alpha}}+\cdots+\eta_{i_{1} i_{2} \ldots i_{k}}^{(k)^{\alpha}}\left(\underset{\sim}{x}, \underset{\sim}{u}, \underset{\sim_{1}^{u}}{u}, \ldots \underset{\sim}{u}\right) \frac{\partial}{\partial u_{i_{1} i_{2} \ldots i_{k}}^{\alpha}}
$$

where

$$
\eta_{i}^{(1) \alpha}=D_{i} \eta^{\alpha}-\left(D_{i} \xi_{j}\right) u_{j}^{\alpha}, \quad i, j=1,2, \ldots, n ; \alpha=1,2, \ldots, m
$$


and

$$
\begin{aligned}
\eta_{i_{1} i_{2} \ldots i_{k}}^{(k)^{\alpha}}=D_{i_{k}} \eta_{i_{1} i_{2} \ldots i_{k-1}}^{(k-1) \alpha}-\left(D_{i} \xi_{j}\right) u_{i_{1} i_{2} \ldots i_{k-1} j}^{\alpha}, \\
\quad i_{l}=1, \ldots, n \text { for } l=1, \ldots, k \text { with } k=2,3, \ldots
\end{aligned}
$$

where $D$ is the total derivative operator defined as

$$
\begin{gathered}
D_{i}=\frac{\partial}{\partial x_{i}}+u_{i}^{\alpha} \frac{\partial}{\partial u^{\alpha}}+u_{i j}^{\alpha} \frac{\partial}{\partial u_{j}^{\alpha}}+\ldots+u_{i_{1} i_{2} \ldots i_{n}}^{\alpha} \frac{\partial}{\partial u_{i_{1} i_{2} \ldots i_{n}}^{\alpha}}+\ldots, \\
u_{i}=\frac{\partial u}{\partial x_{i}}, i=1,2, \ldots, n
\end{gathered}
$$

with summation over a repeated index.

Now, we consider the following Lie group of transformations with independent variables $x, t$; and dependent variable $u$

$$
\bar{x}=\bar{x}(x, t, u ; \varepsilon), \quad \bar{t}=\bar{t}(x, t, u ; \varepsilon), \quad \bar{u}=\bar{u}(x, t, u ; \varepsilon)
$$

where $\varepsilon$ is the group parameter. The infinitesimal generator of BP equation for the Lie group, (4) can be expressed from the formula (3) in the following form:

$$
X=\xi^{x} \frac{\partial}{\partial x}+\xi^{t} \frac{\partial}{\partial t}+\eta^{u} \frac{\partial}{\partial u}
$$

in which $\xi^{x}, \xi^{t}, \eta^{u}$ are infinitesimal functions of group variables (independent and dependent variables).

Theorem 2.1 The BP equation has a three-parameter symmetry group,

$$
X=c_{1} \underset{\sim_{1}}{v}+c_{2} \underset{\sim_{2}}{v}+c_{3} \underset{\sim_{3}}{v}
$$

where

$$
v_{1}^{v}=\frac{\partial}{\partial t}, \underset{\sim_{2}}{v}=\frac{\partial}{\partial x}, \underset{\sim_{3}}{v}=t \frac{\partial}{\partial x}+\frac{\partial}{\partial u}
$$

and, $c_{1}, c_{2}, c_{3}$ are constants and, called group parameters, and also $\underset{\sim_{1}}{v}, \underset{\sim_{2}}{v}, \underset{\sim_{3}}{v}$ are called symmetry generators.

Proof. To calculate the Lie point symmetries of the governing equation (1), first we need to write the third order prolongation of the infinitesimal generator given by (8) since the governing equation include at most third order derivatives. Due to the formula (4), the prolongation of the infinitesimal generator including the related terms is in the following form: 


$$
\begin{gathered}
p r^{(3)} X=\xi^{x} \frac{\partial}{\partial x}+\xi^{t} \frac{\partial}{\partial t}+\eta^{u} \frac{\partial}{\partial u}+\eta_{x}^{u} \frac{\partial}{\partial u_{x}}+\eta_{t}^{u} \frac{\partial}{\partial u_{t}}+\eta_{x x}^{u} \frac{\partial}{\partial u_{x x}} \\
+\eta_{x x x}^{u} \frac{\partial}{\partial u_{x x x}}+\eta_{x x t}^{u} \frac{\partial}{\partial u_{x x t}},
\end{gathered}
$$

where the formulas of terms $\eta_{x}^{u}, \eta_{t}^{u}, \eta_{x x}^{u}, \eta_{x x x}^{u}, \eta_{x x t}^{u}$ are given by the expression (5). Applying the third prolongation of the generator (1) and interacting with mathematica [8], we reach the following determining equations [5-7],

$$
\begin{aligned}
& \left(-u_{x x x} \eta^{u}+\left(1-3 u_{x x}\right) \eta_{x}^{u}+\eta_{t}^{u}-3 u_{x} \eta_{x x}^{u}\right. \\
& \left.-u \eta_{x x x}^{u}-\eta_{x x t}^{u}\right)\left.\right|_{u_{x x t}=u_{t}+u_{x}+u u_{x}-3 u_{x} u_{x x}-u u_{x x x}}=0
\end{aligned}
$$

Calculating the needed terms in (6) and equating every one of the coefficients of independent terms to zero we find the following system of equations after considerable simplifications

$$
\begin{aligned}
& \xi^{x}=\xi^{x}(x, t), \xi^{t}=\xi^{t}(t), \eta^{u}=\eta_{1}^{u}(x, t)+u \eta_{2}^{u}(x, t) \\
& -2 \eta_{2 x}^{u}+\xi_{x x}^{x}=0 \\
& 2 \xi_{x}^{x}-\eta_{2 x x}^{u}=0 \\
& \eta_{2}^{u}+\left(\xi^{t}\right)^{\prime}+\xi_{x}^{x}-6 \eta_{2 x x}^{u}+\xi_{x x x}^{x}=0 \\
& \eta_{1}^{u}+\left(\xi^{t}\right)^{\prime}-\xi_{t}^{x}+\xi_{x}^{x}-2 \eta_{2 x t}^{u}-3 \eta_{1 x x}^{u}+\xi_{x x t}^{x}=0 \\
& \eta_{2 t}^{u}+\eta_{1 x}^{u}+\eta_{2 x}^{u}-\eta_{2 x x t}^{u}-\eta_{1 x x x}^{u}=0 \\
& \eta_{2 x}^{u}-\eta_{2 x x x}^{u}=0 \\
& -\eta_{2 t}^{u}-3 \eta_{1 x}^{u}+2 \xi_{x t}^{x}=0 \\
& -\eta_{2}^{u}-\left(\xi^{t}\right)^{\prime}+\xi_{x}^{x}=0 \\
& \eta_{1 t}^{u}-\eta_{1 x}^{u}-\eta_{1 x x t}^{u}=0
\end{aligned}
$$

¿From (12) it can be seen that the only solution of this over determined system is

$$
\xi^{t}=\alpha_{1}, \xi^{x}=\alpha_{2}+\alpha_{3} t, \eta^{u}=\alpha_{3}\left(\alpha_{1}, \alpha_{2} \text { and } \alpha_{3} \text { arbitrary constants }\right)
$$

which shows that the nonlinear BP equation has only a three-parameter symmetry group

$$
\underset{\sim_{1}}{v}=\frac{\partial}{\partial t}, \underset{\sim_{2}}{v}=\frac{\partial}{\partial x}, \underset{\sim_{3}}{v}=t \frac{\partial}{\partial x}+\frac{\partial}{\partial u} \text { q.e.d. }
$$




\subsection{Optimal system of generators}

A relation between two invariant solutions can be defined to hold true if the first one can be mapped to the other by applying a transformation group generated by a correct linear combinations of the symmetry generators (operators) (9). The relation is an equivalence relation, since it is reflexive, symmetric and transitive, which induces a natural partition on the set of all group invariant solutions into equivalence classes. We need only present one solution from each equivalence class, as the rest may be found by applying appropriate group symmetries; a complete set of such solutions is referred to as an 'optimal system' of group invariant solutions.

The problem of deriving an optimal system of group invariant solutions is equivalent to find an optimal system of generators or subalgebras spanned by these generators (or operators). The method used here is given by Olver in [5], which basically consist of taking linear combinations of the generators (9), and reducing them to their simplest equivalent form by applying carefully chosen adjoint transformations.

Formally, to find an optimal system of one-dimensional subalgebras of $n$ dimensional Lie algebra $L_{n}$, one can separate whole algebra to discrete classes by using equivalent relation

$$
\underset{\sim}{W} \approx \underset{\sim}{W} \Leftrightarrow \exists \varepsilon, \underset{\sim}{W}: \operatorname{Ad}(\operatorname{Exp}(\varepsilon \underset{\sim}{W})) \underset{\sim}{W}=\underset{\sim}{W} \underset{2}{W}
$$

where $(\operatorname{Exp}(\varepsilon \underset{\sim}{W}))$ is the one parameter group of linear transformation which is generated by $\underset{\sim}{W}$ and Ad is the adjoint representation mapping with

$$
\operatorname{Ad}(\operatorname{Exp}(\varepsilon \underset{\sim}{W})) \underset{\sim}{W}=\underset{\sim}{W}-\varepsilon[\underset{\sim}{W}, \underset{\sim}{W}]+\frac{\varepsilon^{2}}{2 !}[\underset{\sim}{W},[\underset{\sim}{W} \underset{\sim}{W}]]-\cdots .
$$

Then the optimal system can be found by choosing one elements from each classes [5].

Theorem 2.2 The optimal system of one dimensional subalgebra of

$$
\begin{aligned}
& L_{3}=\left\{\underset{\sim_{1}}{v}, \underset{\sim_{2}}{v}, \underset{\sim_{3}}{v}\right\} \text { which is Lie algebra admitted by (1) is } \\
& \left\{L_{1,1}(\alpha), L_{1,2}, L_{1,3}, L_{1,4}\right\} \quad \text { where } \\
& L_{1,1}(\alpha \neq 0)=\left\{\alpha \underset{\sim_{1}}{v}+{\underset{\sim}{v}}_{3}\right\} \\
& L_{1,2}=\left\{\tilde{\sim}_{1}\right\} \\
& L_{1,3}=\left\{v_{\sim_{2}}^{v}\right\} \\
& L_{1,4}=\left\{v_{\sim_{3}}^{v}\right\}
\end{aligned}
$$


Proof. First consider the Lie bracket and adjoint representation tables

\begin{tabular}{|c||c|c|c|}
\hline$[]$, & $\mathbf{V}_{1}$ & $\mathbf{v}_{2}$ & $\mathbf{v}_{3}$ \\
\hline \hline $\mathbf{V}_{1}$ & 0 & 0 & $\mathbf{V}_{2}$ \\
\hline $\mathbf{V}_{2}$ & 0 & 0 & 0 \\
\hline $\mathbf{V}_{3}$ & $-\mathbf{v}_{2}$ & 0 & 0 \\
\hline
\end{tabular}

Table 1

Lie Bracket table for $L_{3}$

\begin{tabular}{|c||c|c|c|}
\hline $\mathbf{A d}$ & $\mathbf{v}_{1}$ & $\mathbf{V}_{2}$ & $\mathbf{\sim}_{3}$ \\
\hline \hline $\mathbf{V}_{1}$ & $\mathbf{V}_{1}$ & $\mathbf{v}_{2}$ & $\mathbf{\sim}_{3}-\epsilon \mathbf{\sim}_{2}$ \\
\hline $\mathbf{V}_{2}$ & $\mathbf{V}_{1}$ & $\mathbf{V}_{2}$ & $\mathbf{v}_{3}$ \\
\hline $\mathbf{V}_{3}$ & $\mathbf{V}_{1}+\epsilon \mathbf{\sim}_{2}$ & $\mathbf{V}_{2}$ & $\mathbf{V}_{3}$ \\
\hline
\end{tabular}

Table 2

Adjoint representation table for $L_{3}$

Every elements $\underset{\sim}{v}$ in the Lie algebra $L_{3}$ can be written as $\underset{\sim}{v}=\sum_{i=1}^{3} c_{i} \underset{\sim}{v}$, where $\underset{\sim}{v}$ is given in (9). Without lose of generality, one can choose 3 different cases

case $1: c_{3}=1$

case $2: c_{3}=0, c_{1}=1$

case $3: c_{3}^{2}+c_{1}^{2}=0, c_{1}=1$

and try to find 'the most simple' element from each of them by using (15).

Case 1: The equation

$$
\operatorname{Ad}\left(\operatorname{Exp}\left(\varepsilon{\underset{\sim}{\mathrm{v}}}_{3}\right)\right) \underset{\sim}{W}=\underset{\sim}{W}+c_{1} \varepsilon_{1} \underset{\sim}{v}
$$

for $\underset{\sim}{W}=\sum_{i=1}^{2} c_{i} \underset{\sim_{i}}{v}+\underset{\sim_{3}}{v}$ can be found by using (16). By choosing $\varepsilon_{1}=\frac{-c_{2}}{c_{1}}$, one can set the coefficient of $\underset{\sim_{2}}{v}$ in the right-hand side of (18) to zero, for all $c_{1}, c_{2}$ since $c_{1}$ can never be zero. As a result of this choice, every element of $L_{3}$ with the condition $c_{3} \neq 0$ must be in the some class with $\underset{\sim_{i}}{w^{\prime}}=c_{1} \underset{\sim_{1}}{v}+\underset{\sim_{3}}{v}$. Let's denote $c_{1}=\alpha \in R$ (real numbers). There are two possibilities here

i) $\alpha \neq 0, L_{1,1}=\left\{\alpha \underset{\sim_{1}}{v}+\underset{\sim_{3}}{v}\right\}$

ii) $\alpha=0, L_{1,4}=\left\{{\underset{\sim}{v}}_{3}\right\}$ 
Case 2: By taking $\underset{\sim}{W}=\underset{\sim_{1}}{v}+c_{2} \underset{\sim_{2}}{v}$, one can find the equation

$$
\underline{\sim}^{\prime}=\operatorname{Ad}\left(\operatorname{Exp}\left(\varepsilon_{2}{\underset{\sim}{\mathrm{V}}}_{3}\right)\right) \underset{\sim}{W}={\underset{\sim}{v}}_{1}+\left(c_{2}+\varepsilon_{2}\right) \underset{\sim}{v} .
$$

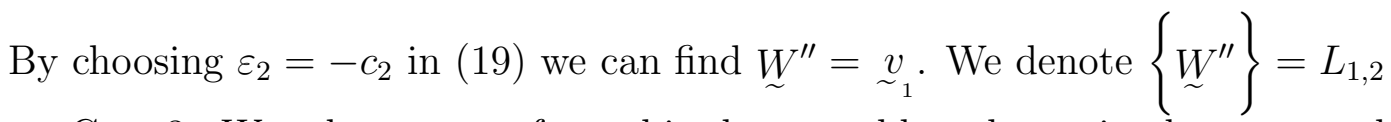

Case 3: We take $\underset{\sim}{W}=\underset{\sim_{2}}{v}$ from this class to add to the optimal system and let's also denote $\{\underset{\sim}{W}\}=L_{1,3}$ q.e.d.

\subsection{Transformation Groups}

Lie group of local point transformations group generated by the vector field $\underset{\sim}{v}=c_{1} \underset{\sim_{1}}{v}+c_{2} \underset{\sim_{2}}{v}+c_{3} \underset{\sim_{3}}{v}$ is

$$
\begin{aligned}
& \bar{x}=\left(c_{2}+c_{1} c_{3} \varepsilon\right) \varepsilon+t c_{3} \varepsilon+x \\
& \bar{t}=c_{1} \varepsilon+t \\
& \bar{u}=c_{3} \varepsilon+u .
\end{aligned}
$$

It is obvious that the transformation (20) transform any solution of (1) of the form $u=u(x, t)$ to function $\bar{u}=\bar{u}(\bar{x}, \bar{t})$ which are solution of (1) itself with $x \rightarrow \bar{x}, t \rightarrow \bar{t}, u \rightarrow \bar{u}$.

\section{Symmetry reductions and group invariant solutions}

In this section, we use the method of characteristics to determine the invariants and reduced ordinary differential equations (ODEs) corresponding to each subalgebra given in (17). Symmetry variables and the invariants of the subalgebras of the Lie algebra $L_{3}$ are given in Table 1 . The result of this can be summarized as follows, where $\xi$ is the symmetry variable, $F(\xi)$ is invariant function related to $u$, and have to be determined using the reduced ODEs. They lead us 4 different types of solutions.

\begin{tabular}{|l|l|l|}
\hline Subalgebra & Symmetry variable & Function $u(x, t)$ \\
\hline$L_{1,1}(\alpha)$ & $\xi=\alpha x-\frac{t^{2}}{2}$ & $u=F(\xi)+\frac{t}{\alpha}$ \\
\hline$L_{1,2}$ & $\xi=x$ & $u=F(\xi)$ \\
\hline$L_{1,3}$ & $\xi=t$ & $u=F(\xi)$ \\
\hline$L_{1,4}$ & $\xi=t$ & $u=F(\xi)+\frac{x}{t}$ \\
\hline
\end{tabular}

Table 1

Invariants of the subalgebras of the Lie algebra $L_{3}$ 


\subsection{Solutions}

3.1.1 $L_{1,1}(\alpha)=\left\{\alpha \underset{\sim_{1}}{v}+\underset{\sim_{3}}{v}\right\}=\left\{\alpha \frac{\partial}{\partial t}+t \frac{\partial}{\partial x}+\frac{\partial}{\partial u}\right\}$

The reduced equation for the subalgebra $L_{1,1}(\alpha)$ is

$$
\alpha^{4} F F^{\prime \prime \prime}+3 \alpha^{4} F^{\prime} F^{\prime \prime}-\alpha^{2} F^{\prime}-1=0 .
$$

The equation (21) can be rewritten as

$$
F^{\prime \prime \prime}=\frac{1}{\alpha^{4} F}+\frac{F^{\prime}}{\alpha^{2} F}+\frac{F^{\prime}}{\alpha^{2}}-3 \frac{F^{\prime}}{F} F^{\prime \prime} .
$$

The equation (22) has a first integral of

$$
W_{F F}^{\prime \prime}= \pm \frac{2}{\alpha^{4} \sqrt{W} F}+\frac{2}{\alpha^{2} F}+\frac{2}{\alpha^{2}}-\frac{3}{F} W_{F}^{\prime}
$$

where $W(F)=\left(F^{\prime}\right)^{2}$.

3.1.2 $L_{1,2}=\left\{{\underset{\sim}{v}}_{1}\right\}=\left\{\frac{\partial}{\partial t}\right\}$

The reduced equation corresponding to $L_{1,2}$ is

$$
F F^{\prime \prime \prime}+3 F^{\prime} F^{\prime \prime}-F F^{\prime}-F^{\prime}=0 .
$$

The general solution of the equation (24) can be given implicitly as

$$
\int_{F} \frac{2 \sqrt{3}|F|}{\sqrt{3 F^{4}+8 F^{3}+12 c_{1} F^{2}-6 c_{2}}} d F=x+c_{3}
$$

where $c_{1}, c_{2}$ and $c_{3}$ are arbitrary constants. By taking $c_{1}=1$ and $c_{2}=0$, a solution of (1) can be found as

$$
u(x, t)=\frac{2}{3}\left(-2+\sqrt{5} \sinh \frac{x}{2}\right) .
$$

3.1.3 $L_{1,3}=\left\{{\underset{\sim}{v}}_{2}\right\}=\left\{\frac{\partial}{\partial x}\right\}$

The reduced equation of $L_{1,3}$ is

$$
F^{\prime}=0
$$

and gives only constant solutions of (1). 
3.1.4 $L_{1,4}=\left\{{\underset{\sim}{v}}_{3}\right\}=\left\{t \frac{\partial}{\partial x}+\frac{\partial}{\partial u}\right\}$

The reduced equation for the subalgebra $L_{1,4}$ is

$$
t F^{\prime}+F+1=0 .
$$

The solution of linear equation (28) is

$$
F=-1+\frac{c_{1}}{t}
$$

where $c_{1}$ is an arbitrary constant. (29) gives a one parameter solution family of (1), as follows

$$
u(x, t)=\frac{x+c_{1}}{t}-1 .
$$

\section{Conclusions}

This paper deals with the symmetry group analysis and classification of the invariant solutions of the Burgers-Poisson equation. In order to obtain exact analytic solution of nonlinear differential equations, the most effective and important approach is the Lie symmetry group analysis. For this purpose, the most general symmetry groups that leave invariant the equation under consideration are investigated. By the standard application of the approach we first prove that the BP-equation has a three-parameter symmetry group. We also give all possible group invariant solutions by using one-dimensional optimal system of Lie symmetry generators of BP-equation in (17), and also the transformation groups which generate those vector fields in (20).

\section{References}

[1] G.B. Whitham, Linear and nonlinear waves, John Wiley \& Sons, New York, 1974 .

[2] K. Fellner and C. Schmeiser, Burgers-Poisson: A nonlinear dispersive model equation, SIAM J.Appl.Math. 64 (2004), 1509.

[3] R. Fetecau and D. Levy, Approximate model equations for water waves, Comm.Math.Sci. 3 (2005), 159.

[4] L.V. Ovsiannikov, Group analysis of differential equations, Moscow:Nauka, 1978 .

[5] P.J. Olver, Application of Lie groups to differential equation, Graduate Texts Math. Vol.10\%, Springer, New York, 1993. 
[6] N.H. Ibragimov, editor. CRC handbook of Lie group analysis of differential equations, Vols. I,II,III, 1994 (English translation, W.F. Ames editor. Published by Academic Press, New York, 1982).

[7] G.W. Bluman and S. Kumei, Symmetries and differential equations, Springer, Berlin, 1989 .

[8] S. Wolfram, Mathematica, Addison-Wesley, California, 1991.

Received: April 17, 2007 